Rev. Saúde públ., S. Paulo

6: 25-33, 1972

\title{
OS NIVEIS DE SAUUDE NA REGIÃO TAQUARI-ANTAS, RIO GRANDE DO SUL, BRASIL
}

João YUNES *

\section{RSPSP-114}

Yunes, J. - Os níveis de sauide na regiấo Taquari-Antas, Rio Grande do Sul, Brasil. Rev. Saúde públ., S. Paulo, 6:25-33, 1972.

Resumo: - Os niveis de saude foram estudados através de uma série histórica (1956-1965), para a Regiấo Taquari-Antas, composta de 50 municípios, com uma população aproximada de $1.300 .000 \mathrm{habi}$ tantes e cerca de $20 \%$ da populaçấo do Estado do Rio Grande do Sul, Brasil. $A$ diminuição do coeficiente de mortalidade geral observada e o aumento da mortalidade proporcional, sugere uma melhora do nivel de saude $e$ isto se observou para as três areas comparadas (Região Taquari-Antas, Estado do Rio Grande do Sul e Pórto Alegre, capital do Estado). A curva de mortalidade proporcional - curva de Nelson de Moraes apresentou a forma de um "J" normal, caracterizando um nivel de saúde regular. Observou-se queda da natalidade para o periodo de estudo, fato que se deve levar em consideraçáo no planejamento da assistência materno-infantil. A mortalidade infantil apresentou decréscimo considerável somente no período 1959. 1960 e, a partir desse ano, até 1965, houve flutuaçóes, não se observando, entretanto, queda ou melhora significativa. As principais causas de bbitos foram as doenças do aparelho circulatório, dos sis. temas nervoso, respiratorio e digestivo. $A$ proporção de $35 \%$ de óbitos classificados como mal definidos, reflete a precariedade da assistência médica na Regiāo. Do total de obitos, 3,3\% foram por moléstias transmissiveis, send̄o que a tuberculose foi responsável por aproximadamente $50 \%$ das mortes por estas causas. A menor proporção de obitos por molestias transmissiveis na Regiấo, quando comparada com a de Pórto Alegre, municipio mais rico do Estado, pode ser rexplicada, em parte, pela grande proporção de óbitos mal definidos e pela migração de pacientes, uma vez que os recursos sanitários disponiveis na região são bastante inferiores aos da Capital do Estado.

Unitermos: - Niveis de saúde*; Estatística vital*; Saúde (diagnóstico).

$$
1 \text { - INTRODUGAO }
$$

O presente estudo integrou o plano de desenvolvimento integrado da Região Taquari-Antas, encomendado pelo Govêrno estadual do Rio Grande do Sul, nos anos de 1969 e 1970 . Duas circunstâncias levaram o govêrno gaúcho a dedicar recursos para o estudo e planejamento des. ta Região. Em primeiro lugar, o fato das vazões de descarga do Rio Taquari-An. tas, dado o seu perfil, serem bastante irregulares e causarem enchentes, com influência mesmo sobre as cheias que afligem Pôrto Alegre, ocasionando pre. juízos substanciais à população e à economia das áreas atingidas. $\mathrm{Em}$ segundo

* Do Centro de Estudos de Dinámica Populacional da Faculdade de Saúde Pública da USP Av. Dr. Armaldo, 715 - São Paulo, SP, Brasil Da Disciplina de Pediatria Social da Faculdade de Medicina da USP - Ay. Dr. Amaldo, 4t5 - São Paulo, SP, Brasil. Da Divisăo de Epidemiologia da Secretaria de Saude do Estado de São Paulo. 
YUNES, J. - Os niveis de saúde na regiåo Taquarl-Antas, Rio Grande do Sul, Brasil. Rev. Saúde pribl., S. Paulo, 6: 25-33, 1972.

lugar, a economia da Região Taquari-Antas passou a apresentar ritmo lento de desenvolvimento. Esta Região, estende-se por cêrca de $45.600 \mathrm{~km}^{2}, 17,0 \%$ da área do Estado do Rio Grande do Sul e possui 50 municípios, com população aproximada de 1.300 .000 habitantes, cêrca de 20,0\% da população do Estado.

Os 50 municípios que integram a Região Taquari-Antas são os que seguem: Anta Gorda, Antonio Prado, Arroio do Meio, Arvorezinha, Barros Cassal, Bento Gonçalves, Bom Jesus, Bom Retiro do Sul, Carlos Barbosa, Casca, Caxias do Sul, Ciríaco, Cruzeiro do Sul, Davi Canabarro, Encantado, Estrêla, Farroupitha, Florres da Cunha, Fontoura Xavier, Garibaldi, General Câmara, Guaporé, Ibiraiaras, Ilipólis, Lajeado, Lagoa Vermelha, Maran, Montenegro, Muçum, Nova Araçá, Nova Bassano, Nova Bréscia, Nova Prata, Paraí, Passo Fundo, Putinga, Rio Pardo, Roca Sales, Salvador do Sul, Santa Cruz do Sul, São Francisco da Penha, São Marcos, Serafina Corrêa, Soledade, Taquari, Triunfo, Vacaria, Venân. cio Aires, Veranópolis.

Para a avaliação dos níveis de saúde, utilizou-se uma série histórica de dez anos, de 1956 a 1965, que permitiu verificar o comportamento dos coeficientes de mortalidade geral, natalidade e mortalidade infantil, bem como as principais causas de óbitos. As séries de informaçóes utilizadas foram até 1965, pois os dados estatísticos que os órgãos oficiais dispunham e em condições de serem submetidos à tabulação se referiam àquele ano. Nesta análise confrontar-se-á a situação da Região com as encontradas no Estado e em sua Capital, Pôrto Alegre.

$$
2 \text { - MORTALIDADE GERAL }
$$

Na região Taquari-Antas, os coeficientes de mortalidade geral sofreram decréscimo no período 1956-1965, conforme Tabela 1 e Figura 1, refletindo certa melhoria das condições sanitárias da área. Os coeficientes, que em 1956 alcançaram o valor de 7,04, decresceram para $5,26 \mathrm{em} \mathrm{1965,} \mathrm{no} \mathrm{total} \mathrm{da} \mathrm{'Região.}$

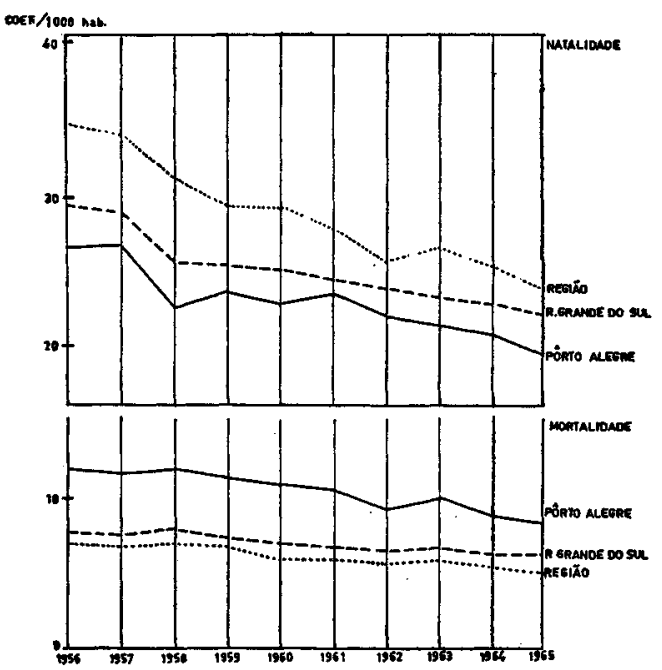

Fig. 1 - Regiño Taquari-Antas - Evolução da natalidade e da mortalidade geral, 1956-1965.

Em todos os anos considerados, os coeficientes de mortalidade na região foram sempre menores do que aquêles verificados em Pôrto Alegre e no Estado. Isto, pode ser explicado pela provável evasão de óbitos para áreas de maiores recursos, sistema de registro de dados, que são efetuados no local de ocorrência e não de procedência, assim como pela influência da diferença da composição da população por sexo e idade. Estas hipóteses são válidas para todos os coeficientes vitais. 
YUNES, J. - Os niveis de saúde na regiåo Taquari-Antas, Rio Grande do Sul, Brasil. Rev. Saúde públ., S. Paulo, 6: 25-33, 1972.

T A B I A 1

Evolução do Coeficiente de Mortalidade Geral na Regiáo Taquari-Antas, em Porto Alegre e no Estado do Rio Grande do Sul* - 1956-1965.

\begin{tabular}{l|c|c|c}
\hline Anos & $\begin{array}{c}\text { Região } \\
\text { Taquari-Antas }\end{array}$ & $\begin{array}{c}\text { Pơrto } \\
\text { Alegre }\end{array}$ & $\begin{array}{c}\text { Estado do } \\
\text { Rio Grande do Sul }\end{array}$ \\
\hline 1956 & 7,04 & 11,96 & 7,83 \\
1957 & 6,58 & 11,83 & 7,70 \\
1958 & 7,14 & 11,90 & 7,93 \\
1959 & 6,87 & 11,35 & 7,65 \\
1960 & 5,92 & 11,06 & 7,14 \\
1961 & 6,00 & 10,70 & 6,92 \\
1962 & 5,76 & 9,75 & 6,50 \\
1963 & 5,94 & 10,05 & 6,84 \\
1964 & 5,57 & 9,61 & 6,44 \\
1965 & 5,26 & 9,24 & 6,34 \\
\hline
\end{tabular}

* Por mil habitantes.

FONTE: Departamento Estadual de Estatística do Rio Grande do Sul.

\section{3 - RAZAO DE MORTALIDADE PROPORCIONAL}

Embora classicamente a taxa de mortalidade proporcional seja calculada a partir de óbitos acima de 50 anos, o índice de Swaroop's \& Uemura2 baseiase nos óbitos acima de 55 anos, por força dos dados divulgados pelo Departamento Estadual de Estatística do Rio Grande do Sul.

A análise da tendência da mortalidade proporcional para a Região, mediante Tabela 2 e Figura 2, comparada com a de Pôrto Alegre e a do Estado, permite observar aumento percentual dos b́bitos acima de 55 anos, o que, de certa maneira, reflete a melhoria do nível de saúde para as três áreas consideradas. Por intermédio desse indicador verifica-se, entretanto, que as condiçōes de saúde da população da Região Taquari-Antas são teoricamente mais satisfatórias que as de Pôrto Alegre e do Estado. As hipóteses explicativas sobre essa distorção são as mesmas apontadas quando da análise da mortalidade geral.

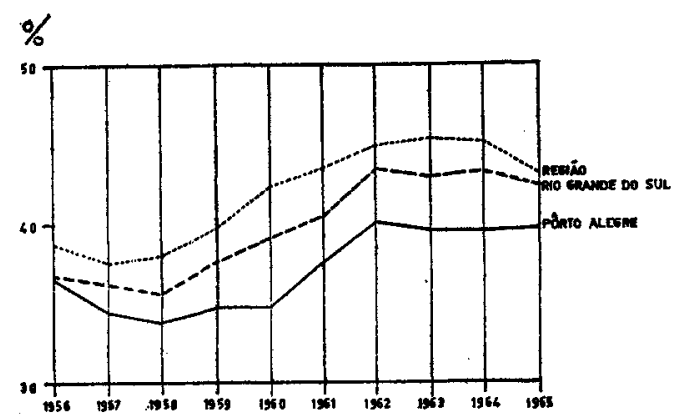

Fig. 2 - Regiăo Taquari-Antas - Evolução da taxa de mortalidade proporcional, 1956-1965. 
YUNES, J. - Os níveis de saúde na regiăo Taquari-Antas, Rio Grande do Sul, Brasil. Rev. Saude pribl., S. Paulo, 6: 25-33, 1972

\section{T A B E L A 2}

Evolução da Mortalidade Proporcional na Regiăo Taquari-Antas, em Porto Alegre e no Estado do Rio Grande do Sul* - 1956-1965.

\begin{tabular}{c|c|c|c}
\hline Anos & $\begin{array}{c}\text { Regtăo } \\
\text { Taquari-Antas }\end{array}$ & $\begin{array}{c}\text { Pôrto } \\
\text { Alegre }\end{array}$ & $\begin{array}{c}\text { Estado do } \\
\text { Rio Grande do Sul }\end{array}$ \\
\hline 1956 & 38,75 & 36,77 & 36,80 \\
1957 & 37,62 & 34,54 & 36,10 \\
1958 & 38,05 & 33,89 & 35,66 \\
1959 & 39,82 & 34,81 & 37,70 \\
1960 & 42,33 & 34,93 & 39,17 \\
1961 & 43,64 & 37,88 & 40,46 \\
1962 & 44,97 & 40,12 & 43,47 \\
1963 & 45,45 & 39,70 & 42,97 \\
1964 & 45,33 & 39,66 & 43,37 \\
1965 & 43,54 & 39,87 & 42,15 \\
\hline
\end{tabular}

* Em percentagem

FONTE: Departamento Estadual de Estatística do Rio Grande do Sul.

\section{4 - CIIRVA DE MORTALIDADE PROPORCIONAL}

Os grupos etários comumente considerados são: menos de um ano; de um a quatro; de cinco a 19 ; de 20 a 49 e 50 anos e mais. Esse indicador demonstra a contribuição de cada um dos grupos etários escolhidos para o total de 6 bitos. De acordo com a forma que a curva assume, é possível avaliar o estágio de saúde de determinada população.

Para compatibilizar a análise com o sistema de coleta de dados do Departamento Estadual de Estatística do Rio Grande do Sul, adotou-se a seguinte divisão para os grupos etários: menos de um ano; de um a quatro; de cinco a 19; de 20 a 54 e 55 anos e mais. A partir dos dados disponiveis, foi construída a cur. va de mortalidade proporcional, Figura 3, que tomou a forma de um "J" normal que, de acordo com Morars", caracteriza nível de saúde regular, tanto para a Região como para Pórto Alegre e para o Estado.

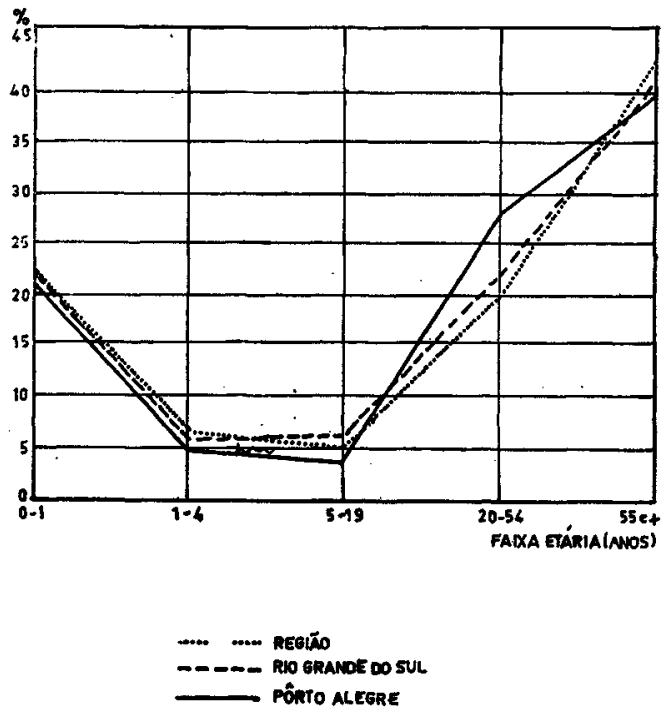

Fig. 3 - Regiåo Taquari-Antas - Curva de mortalidađe proporcional, 1965.

$$
5 \text { - NATALIDADF }
$$

Os coeficientes de natalidade da Região decresceram no período 1956 a 1965 , (Tabela 3 e Figura 1), como também em 
TUNES, J. - Os niveis de saúde na regiăo Taquari-Antas, Rio Grande do Sul, Brasil. Rev. Saude publ., S. Paulo, 6: 25-33, 1972.

TABELA 3

Evolução do Coeficiente de Natalidade na Região Taquari-Antas, em Pôrto Alegre e no Estado do Rio Grande do Sul * - 1956-1965

\begin{tabular}{c|c|c|c}
\hline Anos & $\begin{array}{c}\text { Região } \\
\text { Taquari-Antas }\end{array}$ & $\begin{array}{c}\text { Porto } \\
\text { Alegre }\end{array}$ & $\begin{array}{c}\text { Estado do } \\
\text { Rio Grande do Sul }\end{array}$ \\
\hline 1956 & 35,07 & 26,82 & 29,58 \\
1957 & 34,48 & 27,02 & 29,19 \\
1958 & 31,37 & 22,93 & 25,87 \\
1959 & 29,56 & 23,84 & 25,05 \\
1960 & 29,55 & 23,13 & 25,30 \\
1961 & 28,26 & 23,86 & 24,72 \\
1962 & 25,94 & 22,34 & 24,29 \\
1963 & 26,80 & 21,66 & 23,53 \\
1964 & 25,69 & 21,00 & 23,19 \\
1965 & 24,13 & 19,88 & 22,35 \\
\hline
\end{tabular}

* Por mil habitantes.

FONTE: Departamento Estadual de Estatistica do Rio Grande do Sul.

Pôrto Alegre e no Estado, fenômeno caracteristico, em geral, das áreas em desenvolvimento. Relativamente aos anos limites da série (Tabela 3), os coeficientes apresentaram as seguintes variações: de 35,07 para 24,13, na Regiäo; de 26,82 para 19,88, em Pôrto Alegre; e de 29,58 para 22,35, no Estado. Observese que a natalidade, em 1965, era maior na Região do que em Pôrto Alegre e no Estado, fato que se deve levar em consideração no planejamento da assistência materno-infantil.

\section{6 - MORTALIDADE INFANTIL}

Entre 1956 e 1965, o coeficiente de mortalidade infantil na Região TaquariAntas, conforme Tabela 4 e Figura 4, apresentou decréscimo significativo somente a partir de 1959, passando de 65,40 óbitos por mil nascidos vivos para 52,51, em 1960. A partir dêsse ano e até 1965 houve algumas flutuaçōes, não se verificando, entretanto, queda ou melhoria significativa.

Os coeficientes de mortalidade infantil da Região apresentam-se, paradoxalmente, sempre menores que os do Estado e bem menores ainda que os de Pôrto Alegre, onde não se verificou nenhuma melhoria na década considerada, apesar de concentrar os melhores recursos medico-sanitários do Rio Grande do Sul.

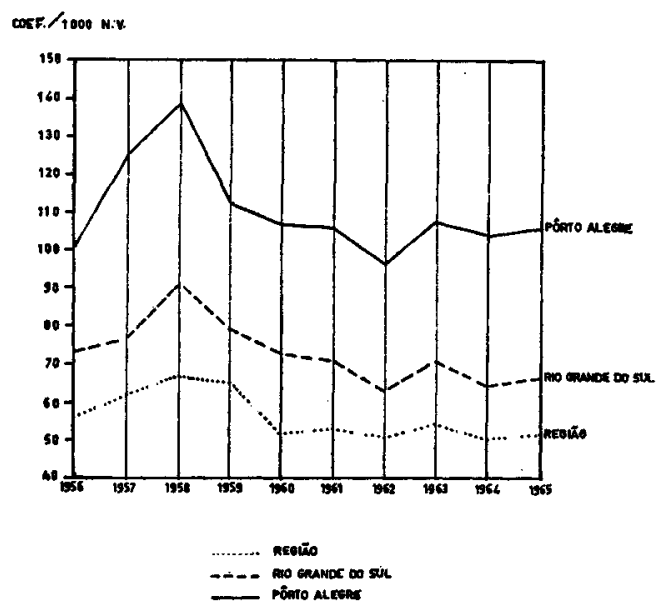

Fig. 4 - Regiăo Taquari-Antas - Evoluğo da mortalidade infantil, 1956-1965.

Em 1965, esse coeficiente oscilou nos 50 municípios que compóem a Região, entre 38,68 e 75,38 . O menor coeficiente mencionado - 38,68 - merece ser des- 
YUNES, $\mathbf{J}$ : - Os niveis de saúde na regiăo Taquari-Antas, Rio Grande do Sul, Brasil. Rev. Saúde públ., S. Paulo, 6: 25-33, 1972.

T A BELA 4

Eroluçăo do Coeficiente de Mortalidade Infantil na Região Taquari.Antas, em Pórto Alegre e no Estado do Rio Grande do Sul * - 1956-1965.

\begin{tabular}{c|cc|c}
\hline Anos & $\begin{array}{c}\text { Região } \\
\text { Taquari-Antas }\end{array}$ & $\begin{array}{c}\text { Porto } \\
\text { Alegre }\end{array}$ & $\begin{array}{c}\text { Estado do } \\
\text { Rio Grande do Sul }\end{array}$ \\
\hline 1956 & 66,76 & 100,50 & 73,02 \\
1957 & 62,34 & 124,19 & 76,06 \\
1958 & 67,01 & 139,09 & 91,64 \\
1959 & 65,40 & 112,77 & 79,79 \\
1960 & 52,51 & 107,00 & 73,15 \\
1961 & 53,89 & 106,55 & 71,19 \\
1962 & 61,85 & 96,23 & 63,08 \\
1963 & 55,69 & 107,89 & 71,38 \\
1964 & 51,27 & 104,63 & 65,37 \\
1965 & 52,71 & 106,53 & 67,90 \\
\hline
\end{tabular}

* Por mil nascidos vivos.

FONTE: Departamento Estadual de Estatística do Rio Grande do Sul.

tacado por não se registrar mesmo em áreas mais desenvolvidas.

Esses fatos permitem formular as seguintes hipóteses, capazes de explicar tal comportamento: má qualidade das informaçōes estatísticas; registro dos obitos nos locais de ocorrência e não nos de origem e sub-registro em áreas mais precárias - zona rural e outras mais afastadas, embora dentro dos perímetros urbanos.

\section{7 - PRINCIPAIS CAUSAS DE óBITOS}

As principais doenças que contribuiram para a mortalidade geral na Região serão analisadas de conformidade com a posição que ocupam em relação ao número total de 6 bitos verificados em 1965. Em ordem decrescente, conforme se verifica na Tabela 5 , destacam-se as doenças da primeira infancia, com 11,9\% dos óbitos totais; neoplasmas, com 8,1\%; doenças do aparelho circulatório, com 7,5\%; doenças do sistema nervoso, com 6,9\%; doenças do aparelho respiratório, com 6,6\%; doenças do aparelho digestivo, com 4,1\%; e doenças infecciosas $e$ parasitárias, com $2 \%$.
O predominio de doenças da primeira infância, que se repete em todo o Estado, reflete a deficiência dos serviços de assistência materno-infantil. Fntre as causas de obito aparecem também enfermidades passiveis de serem controladas mediante programas de medicina preventiva e curativa, como doenças do aparelho digestivo $e$ do aparelho respiratório e as doenças infecciosas e parasitárias, sugerindo um estágio insatisfatório da situação śccio-econômica da Região.

Em Pôrto Alegre, no mesmo ano, verifica-se que as doenças do aparelho respiratório aparecem como a primeira causa de morte, equivalendo a $14,5 \%$ dos obitos. Entretanto, a taxa de $13,5 \%$ para as doenças da primeira infância, também elevada, confirma as deficiências já apontadas.

A elevada participação de $35 \%$ das doenças classificadas como "sintomas de senilidade e causas mal definidas" demonstra a precariedade da assistência médico-sanitária da Região. Para o Estado essa participação é de $32,7 \%$ e, para Pôrto Alegre, de $16,3 \%$. 
YUNES, J. - Os níveis de saúde na regiăo Taquari-Antas, Rio Grande do Sul, Brasil. Rev. Saude puibl., s. Paulo, 6: 25-33, 1972.

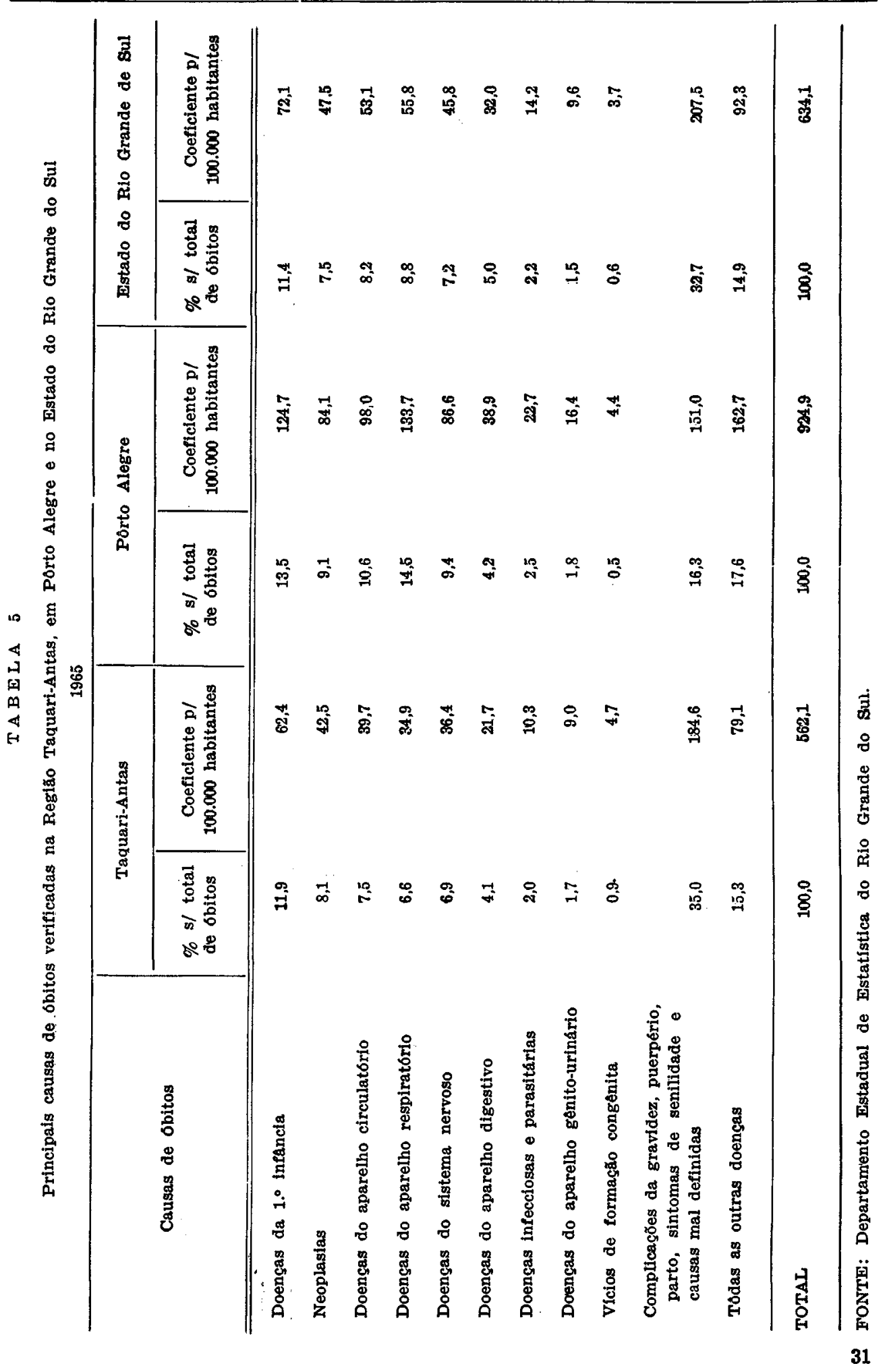


YUNES, J. - Os niveis de saúde na regiåo Taquari-Antas, Rio Grande do Sul, Brasil. Rev. Saride públ., S. Paulo, 6: 25-33, 1972.

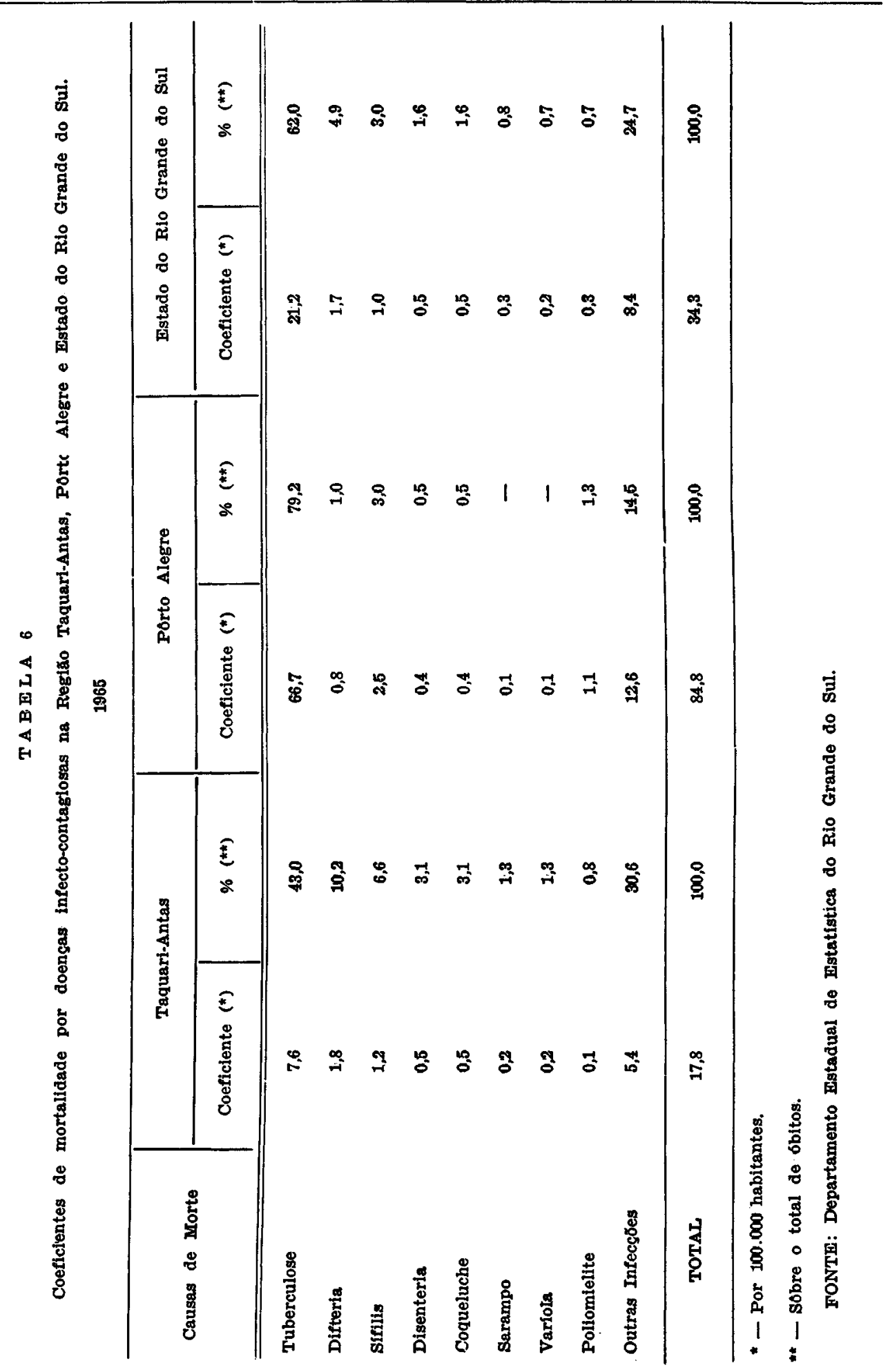


YUNES, J. - Os niveis de saúde na região Taquari-Antas, Rio Grande do Sul, Brasil. Rev. Saúde públ., S. Paulo, 6: 25-33, 1972.

Entre as principais moléstias transmissiveis, responsáveis por 3,3\% dos obitos gerais na Região destacam-se, por ordem decrescente, a tuberculose, com $43,0 \%$ dos óbitos; difteria, com $10,2 \%$; sífilis, com $6,6 \%$; disenteria, com $3,1 \%$; coqueluche, com $3,1 \%$; e varíola com 1,3\%. (Tabela 6).

Esses óbitos, da mesma forma considerados teoricamente evitáveis, demonstram que também nessa classe de enfermidades, a assistência médico-sanitária regional é deficiente.

A Região Taquari-Antas apresenta, como se disse, $3,3 \%$ de óbitos por moléstias infecto-contagiosas, enquanto que no Estado essa proporçäo é da ordem de $5,4 \%$ e, em Pôrto Alegre, de 9,2\%.

A menor mortalidade proporcional por moléstias transmissíveis verificada na Região, explica-se pela grande percentagem de óbitos mal definidos e pela migração de pacientes, uma vez que os recursos sanitários disponíveis na área são bastante inferiores aos de Pôrto Alegre.

RSPSP-114

Yunes, J. - [Health levels in the Taquari-Antas Region, State of Rio Grande do Sul, Brazil.] Rev. Saúde públ., S. Paulo, 6:25-33, 1972.

Summary: - Health levels were studied through a historical series (1956-1965), in the Taquari-Antas region (State of Rio Grande do Sul - Brazil), composed of 50 cities with a population of nearly 1300000 inhabitants, about $20.0 \%$ of the population of the State. The fall of crudie death rate and the increase of the mortaly ratio (above 55 years old) suggests $a$ better improvement on the health le- vels and this trends was showed in the three compared areas (Taquari-Antas region, State of Rio Grande do Sul and Porto Alegre the capital of the State). Nelson de Moraes' Curve (proportional death ratio by age group) showed a normal form of a " $J$ ", which demonstrated a regular health level of the studied area. The birth rate declined in the studied period of time. This fact is worth being considered in the maternal and child health planning. The infant mortality rate showed a decreasing in the 1959-60 years and from this time up to 1955 there was a fluctuation. However, a significative decrease or improvement was not observed. The main causes of death were Circulatory, Nervous System, Respiratory and Digestive System diseases. The ratio of $35 \%$ of death was classified as "ill defined" which means a lack of medical cane in the studied region. From the total death, 3.3\% were due to communicable disease, and among these diseases tuberculosis was responsible for about $50.0 \%$ of the death. When compared with Porto Alegre, the richest city of the State of Rio Grande do Sul, the lower proportion of death for communicable disease in the studied nogion can be explained in part due to the proportion of death classified as "ill defined" and to the migration of patients, since the health medical care resources in the studied area are lower than that one compared with the city of Porto Alegre.

UNITERMS: - Health levels"; Vital statistics*.

REFERENCIAS BIBIOGRAFICAS

1. Moraes, N. L. de A. - Níveis de saúde de coletividades brasileiras. Rev. Serv. Saúde públ. (Rio de J.), 10:408-97, 1959.

2. SWAROOP, S. \& UEMURA, K. - Proportional mortality of 50 years and above: a suggested indicator of the component health, including demographic conditions in the measurement of levels of living. Bull. Wld Hlth Org., 17:439-81, 1957.

Recebido para publicagão em 29-11-1971

Aprovado para publicacáo om 7-1-1972 